\title{
LA RESPUESTA DE LAS NIÑECES, ADOLESCENCIAS Y JUVENTUDES DEL COLECTIVO SIN FRONTERAS FRENTE AL CONTEXTO CONTINUO DE CRISIS SOCIOPOLÍTICA Y SANITARIA ${ }^{1}$
}

\author{
EDUARDO GALO ERAZO ${ }^{2}$ \\ NADIME MASRI ORTUÑO ${ }^{3}$ \\ GABRIEla PANES ÁlVAREZ ${ }^{4}$ \\ JUAN ZAMORANO HERNÁNDEZ ${ }^{5}$
}

\begin{abstract}
RESUMEN
Este artículo viene a develar, problematizar y visibilizar las distintas respuestas de las niñeces, adolescencias y juventudes (NAJ) ${ }^{6}$ pertenecientes a la Corporación Colectivo Sin Fronteras frente a un contexto de crisis provocada por la llegada del Covid-19. A través de una sistematización de dos años que rescata una serie de hitos que dan cuenta de la capacidad de asociatividad y agencia mediante espacios de encuentro basados en la afectividad, la colaboración, el respeto, la solidaridad y buenos vivires que se materializan en acciones de promoción y exigibilidad, interpelando a diferentes garantes de derechos, actores sociales y políticos cuyas decisiones repercuten en la vida cotidiana de las NAJ.

PALABRAS CLAVE: NIÑECES, ADOLESCENCIAS Y JUVENTUDES (NAJ), CRISIS SOCIOPOLÍTICA Y SANITARIA, ACCIÓN COLECTIVA, CAPACIDAD DE AGENCIA, BUENOS VIVIRES.
\end{abstract}

${ }^{1}$ Este artículo responde a un trabajo de sistematización realizado desde abril de 2019 hasta la fecha.

${ }^{2}$ Educador/Sociólogo de la Universidad de Toulouse II, Magíster en Ciencia Política mención Gobierno y Políticas Públicas de la Pontificia Universidad Católica de Chile. Correo electrónico: ejgalo@uc.cl

${ }^{3}$ Educadora/Psicóloga, Magíster en Psicología Social Jurídica de la Universidad Nacional Andrés Bello. Psicóloga educacional. Correo electrónico: nadimefmasri@gmail.com

${ }^{4}$ Educadora/Terapeuta Ocupacional de la Universidad Andrés Bello, Docente de la Universidad Andrés Bello. Fundadora del Centro Cultural y Comunitario La Minga. Correo electrónico: gabrielapanesalvarez@gmail.com

${ }^{5}$ Educador/Terapeuta Ocupacional de la Universidad Andrés Bello. Magíster(c) en Ciencias Sociales, mención Estudios de la Sociedad Civil, Universidad de Santiago de Chile. Docente de la carrera de Terapia Ocupacional, USACh. Coordinador del Área de Promoción de Derechos de las Niñeces y Adolescencias, Corporación Colectivo Sin Fronteras. Correo electrónico: jzamorano@sinfronteraschile.cl

${ }^{6}$ El presente escrito utilizará el concepto de $N A J$ para referirse a los niños, niñas, niñes, adolescencias y juventudes con la finalidad de validar las distintas identidades con las cuales se reconocen. 


\title{
A RESPOSTA DAS CRIANÇAS, ADOLESCENTES E JOVENS DO COLECTIVO SIN FRONTERAS NO CONTEXTO CONTÍNUO DE CRISE SOCIAL, POLÍTICA E SANITÁRIA
}

\begin{abstract}
RESUMO
Este artigo revela, problematiza e torna visíveis as diferentes respostas das crianças, adolescentes e jovens $(N A J)$ pertencentes à Corporação Colectivo Sin Fronteras no contexto de crise provocado pela chegada da Covid-19. Através de uma sistematização de 2 anos, foram identificados uma série de acontecimentos que mostram a capacidade de associatividade e agência mediante espaços de encontro baseados na afetividade, a colaboração, o respeito, a solidariedade e o bom viver que se materializam em ações de promoção e exigibilidade, desafiando diferentes garantidores de direitos, atores sociais e políticos cujas decisões têm um impacto na vida cotidiana das $N A J$.

PALAVRAS-CHAVE: NAJ, CRISE SOCIOPOLÍTICA E SANITÁRIA, AÇÃO COLETIVA, CAPACIDADE DE AGÊNCIA, BOM VIVER.

\section{THE RESPONSE OF CHILDREN, ADOLESCENTS, AND YOUNG PEOPLE FROM COLECTIVO SIN FRONTERAS TO THE CONTINUOUS CONTEXT OF SOCIO-POLITICAL AND SANITARY CRISIS}

\begin{abstract}
This article reveals, problematizes, and evidences the different responses of children, adolescents, and young people $(N A J)$ from the Colectivo Sin Fronteras Corporation to the context of crisis caused by the arrival of COVID-19. Through a 2-year systematization of a series of landmarks showing the capacity of associativity and agency through spaces of encounter based on affectivity, collaboration, respect, solidarity, and good living materialized in actions of promotion and enforceability, questioning the different rights guarantors, and social and political actors whose decisions have an impact on the daily life of $N A J$.
\end{abstract}

\footnotetext{
KEYWORDS: $N A J$, SOCIO-POLITICAL AND SANITARY CRISIS, COLLECTIVE ACTION, AGENCY CAPACITY, GOOD LIVING.
} 


\section{INTRODUCCIÓN}

En el año 1990, Chile suscribe y ratifica la Convención sobre los Derechos del Niño $(\mathrm{CDN})^{7}$, un tratado de índole internacional adoptado por la ONU que reconoce y protege los derechos de las $N A J$ (ONU, 1989). La CDN forja un tratado a nivel internacional en materia de niñez y derechos humanos debido a la necesidad de implementar normas de protección a las $N A J$ que respondan a sus necesidades en situaciones adversas en donde sus derechos se vean vulnerados. Ante esta situación se identifican tres principales garantes de derechos ${ }^{8}$ : el Estado, la sociedad civil y la familia, siendo estas las entidades responsables de velar por el bienestar integral de la niñez, propiciando el pleno ejercicio de sus derechos. Es así que la CDN sostiene cuatro principios fundamentales que orientan a la implementación del goce pleno y promoción de los derechos, los que refieren a: la no discriminación, interés superior de la niñez, garantía de la supervivencia y el pleno desarrollo, y la participación infantil (Valverde, 2004). De esta manera, los Estados parte asumen responsabilidades políticas con y para las $N A J$ que deben ser materializadas y visibilizadas a través del acceso y alcance a sus derechos fundamentales, roles y espacios que tienen en la sociedad.

En el caso de Chile, la garantía del pleno acceso a los derechos para las $N A J$ dista en gran medida de la realidad que vivimos actualmente, estando desprotegidas al momento de ver vulnerados sus derechos fundamentales cuando el Estado reconoce un rol subsidiario en su promoción, implementación y protección de estos. Es posible identificar esta desprotección en la imposibilidad

7 Es importante señalar que previo a la CDN, la historia oficial reconoce las declaraciones de «Moscú» (1918) y «Ginebra» (1924), así como la declaración de los derechos del niño (1959) como instrumentos internacionales por la protección de los derechos de las niñas y niños (Liebel, 2016; Morales y Magistris, 2018).

${ }^{8}$ Entendidos como aquellas instituciones responsables de crear/generar condiciones de respeto y ejercicio de los DD.HH. de todas las personas, sin distinción alguna. 
que tienen las $N A J$ de hacer libre ejercicio a sus derechos - familia, cuidado, educación, salud, vivienda, recreación, opinión, entre otros-, ya sea por los múltiples sistemas de opresión que se hacen presentes en aquellas relaciones humanas y materiales que se encuentran al momento de referirnos a los derechos, como también las situaciones de vulneración a niveles inter e intrapersonales. Dentro de estos sistemas, es posible reconocer las consecuencias del colonialismo, neoliberalismo, patriarcado y adultocentrismo, entre otros tipos de violencia, como claves para la realidad que hoy se performa en torno a la vida cotidiana de las $N A J$ a lo largo del país. Generalmente, las realidades conocidas por las $N A J$ se ven caracterizadas por el abandono y desamparo estatales, espacios educativos adultistas ${ }^{9}$ ritmados por dinámicas de discriminación y situaciones constantes de bullying ${ }^{10} \mathrm{o}$ acoso escolar.

Estas realidades posiblemente se tornan con mayores dificultades cuando atraviesan situaciones migratorias a los sistemas ya señalados. Es así como es posible ejemplificar, a través del «Plan de Retorno Voluntario», el rechazo y nulo reconocimiento del Pacto Migratorio Mundial y la actual ley migratoria como hitos que vienen a retratar las formas en que se piensa gubernamentalmente la migración, desarrollando visiones securitarias que suelen pasar a llevar los derechos de las personas migrantes.

\footnotetext{
${ }^{9}$ Entendiéndose como el acto de discriminación por edad, es la materialización de la violencia cuya lógica se encuadra en el adultocentrismo (Alexgaias, 2014) (para que se entienda mejor, si hacemos un paralelismo, el adultocentrismo es al patriarcado o al colonialismo, lo que el adultismo es al machismo o al racismo).

${ }^{10}$ Bullying apuntando a la confrontación de los intereses o necesidades en que ciertos protagonistas adoptan un rol dominante y obligan por la fuerza a que otro se ubique en un rol de sumisión, causándole con ello un daño que puede ser físico, psicológico, social o moral (Gómez Nashiki, 2013).
} 
De esta manera, recientemente el Comité de Trabajadores Migrantes de la $\mathrm{ONU}^{11}$, los relatores especiales y expertos independientes nombrados por el Consejo de Derechos Humanos de la ONU señalan que las deportaciones que se han dado en Chile desde febrero de 2021 han resultado en la deportación de cientos de migrantes sin una evaluación individual ni la posibilidad de solicitar protección, de acuerdo con el derecho internacional de derechos humanos y refugiados. Ante esta situación, la Defensoría de la Niñez ${ }^{12}$ indica que al realizar expulsiones masivas de migrantes, el interés superior de las $N A J$ migrantes y su derecho a vivir en familia no están siendo respetados, por lo que la CDN y sus principios estarían viéndose transgredidos.

Hablamos así de vulneraciones en que la violencia adquiere ciertas particularidades propias a los procesos migratorios, en donde la exclusión toma fuerza e impacta a las $N A J$ en todas las esferas de sus vidas (Fundación Friedrich Ebert-Chile, 2017). En otras palabras, estamos frente a la articulación de una serie de sistemas de opresión que continúan perpetuando la precarización de la vida.

Es a raíz de lo anterior, que es posible señalar que el contexto de crisis dentro del Colectivo Sin Fronteras se reconoce como una realidad previa a la revuelta del 18 de octubre de 2019, siendo este hecho un destape de las diferentes vulneraciones que se estaban viviendo en los distintos territorios. Durante este periodo es posible destacar que las $N A J$ no solo fueron iniciadoras de este proceso social, sino que a su vez fueron víctimas de las diferentes violencias sistemáticas perpetradas por agentes del Estado hacia quienes ejercen su libre derecho a la expresión y manifestación (Defensoría de la Niñez, 2020). Ante esto las

\footnotetext{
${ }^{11}$ Cristián Campos (2021, 19 de mayo). "Arbitrary expulsions of migrants in Chile must stop immediately, urge rights experts". UN News. Disponible en: https://news.un.org/en/story/2021/05/1092282

12 Disponible en: https://www.defensorianinez.cl/noticias/expulsiones-masivas-demigrantes-defensora-de-la-ninez-llama-a-poner-en-el-centro-interes-superior-deninos-ninas-y-adolescentes-migrantes-y-su-derecho-a-vivir-en-familia/
} 
respuestas se enmarcan en torno a la violencia a través de represión en la calle, en donde los golpes, balines, abusos, hostigamientos en poblaciones, barrios, hogares, detenciones arbitrarias, entre otras situaciones, se hacen presentes.

Es así que se develan las desprotecciones existentes por parte del Estado y sus instituciones, permeando en las realidades de las familias en sus diferentes esferas. Sin embargo, estas situaciones de precariedad y abandono se vieron intensificadas ante la llegada de la pandemia del SARS-CoV-2, en donde la desprotección y vulneración de derechos de las $N A J$ se vieron profundizadas.

Es debido a lo anterior que urge señalar y reconocer estas prácticas sistemáticas e históricas como malos vivires ${ }^{13}$, siendo esta la causa de un malestar colectivo permanente (Montero, 2004). Ante esto nos preguntamos: ¿cuál es la respuesta que las $N A J$ desarrollan frente al contexto continuo de crisis sociopolítica y sanitaria? Interrogante que se responderá y analizará a partir de la experiencia de las $N A J$ pertenecientes al Colectivo Sin Fronteras, que se presenta a continuación.

\section{El Colectivo Sin Fronteras}

La Corporación de Investigación, Desarrollo de la Sociedad y las Migraciones, «Colectivo Sin Fronteras» (CSF), es una organización que desde el año 2003 trabaja por la interculturalidad, la inclusión social y los derechos de las $N A J$ y familias que son parte de los movimientos migratorios que se vienen asentando en territorio chileno en las últimas décadas, siendo especialmente las $N A J$ socioeconómicamente más oprimidas su principal preocupación. Las

\footnotetext{
${ }^{13}$ Entenderemos malos vivires como el resultado de la convergencia de las opresiones, violencias y priorizaciones de la vida ya señaladas, que performan una realidad que impacta negativamente en ámbitos físicos, psicológicos, económicos, políticos y espirituales.
} 
familias que se relacionan con el CSF provienen de países como Perú, Ecuador, Bolivia, Colombia, Argentina, Haití, Venezuela y República Dominicana, así como un número importante de chilenas y chilenos que pertenecen a la Comunidad Sin Fronteras ${ }^{14}$.

Su misión, durante todos estos años, ha sido promover la convivencia intercultural entre la población nacional y migrante, así como el ejercicio de derechos de las $N A J$ y sus familias que han migrado a Chile. Por tanto, su visión y esfuerzos están centrados en constituir con todos los actores de la sociedad civil (nacional y migrante), una sociedad que cuente con condiciones legales, sociales y culturales que garantice los derechos de las $N A J$ y sus familias ${ }^{15}$ en situación de migración.

Sus acciones comenzaron con un stand informativo en la Plaza de Armas de Santiago, durante los años 2003-2005, acompañando principalmente a familias provenientes de Perú en sus procesos de regularización migratoria hasta tener la posibilidad, en la actualidad, de desarrollar su apuesta política en una casa colectiva (sede central) ubicada en el barrio La Chimba ${ }^{16}$, específicamente en la calle Barnechea \#320, comuna de Independencia.

El CSF ha buscado desarrollar su trabajo de promoción de derechos y de interculturalidad en diferentes territorios con alta presencia de población migrante, teniendo actualmente espacios establecidos en las comunas de Independencia (casona Barnechea, casona Maruri y Espacio Bustos), Santiago (espacio Almirante Latorre y casona Yungay) y Quinta Normal (La Junta). Como

\footnotetext{
${ }^{14}$ La Comunidad Sin Fronteras se compone actualmente por un total de 108 familias, siendo 164 Niñeces y Juventudes las que acompaña el CSF.

${ }^{15}$ Disponible en: https://www.sinfronteraschile.cl/

${ }^{16}$ Del quechua «de la otra banda»; «del otro lado del río», haciendo alusión al río Mapocho.
} 
también, colaborando y manteniendo vínculos con organizaciones en los territorios de Lo Prado, Estación Central y Recoleta.

\subsection{Propuesta política del CSF}

La propuesta política del CSF nace como respuesta de la sociedad civil, apostando por la inclusión social y la interculturalidad, develando lo que significa encontrarse en situación de migración en un país como Chile. Además, el trabajo con las $N A J$ se ha visibilizado y legitimado, siendo reconocido como una experiencia única en el contexto chileno, que ha puesto los derechos y el protagonismo de las $N A J$, así como la interculturalidad, como parte de las preocupaciones de sectores organizados de la sociedad civil y en parte de la agenda política, posicionando a las $N A J$ como interlocutores válidos ${ }^{17}$ en el espacio público (Morales y Magistris, 2018).

Por lo mismo, el proyecto político del CSF se desarrolla desde ahí con el objetivo de dignificar la vida, visibilizando la importancia de identificar cuáles son los derechos de las $N A J$ y quiénes son los actores y entidades responsables de garantizarlos (Valverde, 2004).

El desarrollo de esta propuesta viene a tensionar las formas en que concebimos la sociedad y nos invita a relacionarnos desde el respeto y reconocimiento de las culturas, el buen trato, la no discriminación y el rol protagónico de las niñeces al momento de repensar nuestro lugar en el tejido social que habitamos. El hecho de comprendernos como una comunidad en un contexto de crisis significa dar respuesta ante las necesidades que han existido incluso antes de la crisis sociopolítica y sanitaria, que ha venido a profundizarlas

\footnotetext{
${ }^{17}$ Luego de sus primeros años de experiencia, el CSF evidenció la necesidad de trabajar con el grupo más golpeado dentro de los flujos migratorios, las niñeces y juventudes, destacando su rol protagónico, con capacidad de incidir en la esfera pública y en las decisiones que les atañen.
} 
aún más.

A partir de este contexto de vulneración, el CSF ha diseñado una serie de estrategias de promoción y restitución de sus derechos, siguiendo los lineamientos planteados en su marco conceptual desde perspectivas críticas; esto quiere decir que orienta su praxis hacia la transformación social, facilitando y fortaleciendo los procesos psicosociales que posibilitan el desarrollo de comunidades autogestionadas en la solución de sus problemas (Montero, 2004). Por lo mismo, se posiciona a partir de diferentes enfoques y líneas teóricas que van en la línea de dichas modificaciones estructurales, tales como el enfoque de interculturalidad crítica, evidenciando que el problema no surge desde la diferencia o diversidad cultural, sino que desde la raíz estructural-racial-colonial (Walsh, 2010).

Para efectos de este artículo se entiende la interculturalidad «como una herramienta, como un proceso y proyecto que se construye desde la gente - $-\mathrm{y}$ como demanda de la subalternidad (...) requiere la transformación de las estructuras, instituciones y relaciones sociales, y la construcción de condiciones de estar, ser, pensar, conocer, aprender, sentir y vivir distintas» (p. 78). En este sentido, la interculturalidad entendida críticamente aún no existe, es algo por construir (Walsh, 2009). Por eso, se entiende como una estrategia, acción y proceso permanente de relación y negociación entre las diferentes culturas, en condiciones de respeto, legitimidad, simetría, equidad y reciprocidad (Walsh, 2010).

En esta misma línea, se utiliza el enfoque de participación protagónica, el cual hace referencia a una participación activa, total, libre y consciente, que comprende y reconoce la propia dignidad (Cussianovich y Márquez, 2002; Save the Children Suecia, 2008) y la concepción de igualdad entre las $N A J$ y adulteces. Para esto, es imprescindible la deconstrucción de las actuales representaciones sociales que se tienen de la niñez, ampliando la mirada hacia otras que consideran 
a $N A J$ como actores sociales y políticos, comprendiendo así la participación como un ejercicio de actoría social y de construcción de identidades (Cussiánovich y Márquez, 2002), reconociendo que tanto adulteces como las NAJ pueden ejercer su ciudadanía, y por tanto, son sujetos de derechos.

A su vez, a través del enfoque de género el CSF visibiliza las asimetrías de poder producidas desde lógicas colonialistas y patriarcales construidas socialmente, así como los roles, expectativas y formas de opresión propios de la masculinidad hegemónica (Facio, 2005). Opresión que no solo viven las mujeres, niñas y niñes, sino que también hombres y niños producto de los mandatos y construcciones relacionados con el género (Obach, Sadler y Aguayo, 2011).

Por último, el CSF se sitúa desde un enfoque de derechos humanos, que aparece de manera transversal en cada una de sus acciones, enfatizando en que las personas se convierten en sujetxs ${ }^{18}$ de derechos por el solo hecho de ser personas. Este enfoque, además, reconoce la ciudadanía de todas las personas sin distinción en el marco del derecho a una democracia participativa, en la cual las relaciones interpersonales se dan desde la equidad y el respeto a las diferencias, enfatizando que las personas son sujetxs integrales con derechos universales e indivisibles (Solís, 2003). Lo anterior toma relevancia en el sentido de la adquisición de una conciencia de las propias responsabilidades y derechos, ante todo en la medida en que son sometidos a un sistema de reglas y atribuciones sociales que les otorga identidad social (Save the Children Suecia, 2008). Por ende, a las $N A J$ — desde su lugar como actores estratégicos y políticos- se les reconoce como parte fundamental para las distintas expresiones que atraviesa la sociedad y cultura, siendo vital su participación colectiva para impulsar procesos

${ }^{18}$ En esta publicación usaremos la equis ( «X») como forma de nombrar todos los géneros: varones, mujeres y todas aquellas identidades que no formen parte del esquema binario hegemónico. Reconocemos la complejidad de su lectura, sin embargo, así como hacemos esfuerzos para repensar la niñez, también invitamos a repensar el lenguaje y en cómo incluir a cada quien. 
de cambios y transformaciones sociales ${ }^{19}$.

Todos estos enfoques responden a lo que entenderemos por buenos vivires $^{20}$.

\subsection{PrinCIPIOS METODOLóGICOS}

Es así como los buenos vivires apuestan por una lógica y visión de mundo que viene a exigirnos reflexión y análisis sobre nuestras dinámicas sociales y los roles que vamos adquiriendo en este tejido social. Es por esto que la ciudadanía crítica y radical viene a ser un principio metodológico fundamental (González, 2015), lo que para efectos de este artículo la comprendemos como el reconocimiento de la ciudadanía de todas las personas superando los edificios jurídicos actuales, centrados en «un enfoque excluyente y en una noción de ciudadanía tan abstracta como estandarizante» (Güendel, 2000: 174). Por lo mismo comprendemos a las $N A J$ como sujetxs de derechos, como ciudadanxs activxs que deben tener la posibilidad de incidir desde su lugar, para ser parte de las discusiones en los espacios públicos y privados, es decir, «la ciudadanía es más que el derecho a voto y que implica pensar el modo de relación entre niños y niñas y las estructuras sociales, organizadas desde el mundo adulto» (González, 2015). Esto nos permite democratizar ciertas áreas antes excluidas de la discusión pública y la aparición de nuevos protagonismos de las $N A J$, quienes pasan a ser parte de la construcción de una comunidad política en la que sí son reconocidas. El hecho de comprender a las $N A J$ desde un enfoque de derechos humanos, nos

${ }^{19}$ Rescatando las movilizaciones de Chile en las últimas décadas, principalmente estudiantiles, tales como: Mochilazo (2001), Revolución Pingüina (2006), Movilización Estudiantil (2011), Mayo Feminista (2018), Salto del Torniquete (2019).

${ }^{20}$ El Buen Vivir es la formulación más antigua de la resistencia indígena frente a la colonialidad del poder (Quijano, 2011) y la reafirmación de la existencia de un modo de vida diferente al occidental. 
hace distinguir y resaltar su capacidad de asociatividad, autogobierno, autogestión, autodeterminación y agencia particular.

Las $N A J$ transitan gran parte de su cotidiano en un contexto educativo específico que se encuentra permeado por lógicas estructurales tradicionales, que a través de un proceso de institucionalización, reduce el significado del vínculo educativo a la idea del educador como alguien que deposita ciertos contenidos al estudiante, entendiendo esta como una educación bancaria, asimétrica (Areyuna, Cabaluz y Zurita, 2018) y adultista (Cussianovich, 2011). Por tanto, urge poder mirar alternativas pedagógicas transformadoras que se basen en el reconocimiento de la otra persona, validando los sentires, emociones y saberes de las $N A J$, pudiendo resignificar la importancia de las relaciones socioafectivas que crean, desarrollan y sustentan constantemente a una comunidad educativa, lo que llamaremos ternura. Esta pedagogía de la ternura la comprenderemos como «la capacidad de afectividad respetuosa, en una relación marcada por el componente emocional y afectivo, por los sentimientos como una manera de establecer un vínculo emancipador desde una mirada crítica y política que lleva a transformar» (Cussianovich, 2010). Así es como dicha pedagogía viene a ser una alternativa educativa transformadora que se encuentra bajo el manto de las subjetividades y la validación de sus emociones. Por consiguiente, el quehacer pedagógico permite nuevas formas de entender la educación desde una mirada integral, libre, emocional, crítica y reflexiva de los contextos donde las $N A J$ se desarrollan.

Comprender la educación como un fenómeno transformador que se encuentra sustentado en términos políticos, nos exige analizar dichas alternativas pedagógicas invitándonos a concientizar los procesos de los que somos parte. La pedagogía de la liberación la entenderemos como «una pedagogía para la transición social, y por tanto define su actividad educativa como una acción cultural cuyo objetivo central puede resumirse en el término concientización» (Torres, 2002: 36-37). Así es como a través del tiempo se fueron identificando 
ejes a trabajar en el ámbito educativo que pasaron a ser exigencias de la sociedad civil organizada, como lo es la educación no sexista, educación popular y la educación cívica, identificando los intereses de aprendizaje desde una perspectiva crítica, propiciando espacios de encuentro y reflexión que se performan en el tiempo, validando la importancia del vínculo socioafectivo, sentires y emocionalidad de las $N A J$ (Freire, 2005).

Es de vital importancia visibilizar el rol político y social que van tomando las $N A J$ en sus propias realidades, ya que son actores sociales postergadxs y oprimidxs que deben ser protagonistas de sus propios procesos, donde se fortalezca su autodeterminación, destacando el concepto de coprotagonismo, siendo un principio fundamental y una necesidad ética, enmarcada en «una teoría crítica de las teorías de infancia»(Cussiánovich, 2011: 38). Es así como el coprotagonismo nos interpela e invita a cuestionar el adultocentrismo, y caminar hacia la construcción de un nuevo modo de entablar las relaciones sociales intergeneracionales. Según esta perspectiva, la participación aloja en sí un salto cualitativo al incidir en el proceso del ejercicio del conjunto de los derechos, colocando a las $N A J$ como actores sociales políticxs activxs en sus diferentes espacios cotidianos de participación (Alfageme et al., 2003).

\subsection{PRESENTACIÓN DE LOS ESPACIOS}

Los principios metodológicos se traducen en el trabajo directo con las $N A J$ por medio de dos programas: Escuelita Libre Sin Fronteras (ELSF) y los Colectivos de Niñeces y Adolescencias en Acción (CNAA), cuyo objetivo es que las $N A J$ demanden atención de sus vulneraciones de derechos y trabajen activamente por mejorarlos.

En la ELSF destacan los talleres de Apoyo Escolar, El Taller Aprendiendo Sin Cuadernos (TASC), el Acompañamiento Personalizado y el Taller de 
Adolescencias, los cuales van en la línea de acompañar el derecho a la educación (y otros derechos), además de que las $N A J$ cuenten con procesos de escolaridad estables basados en el apoyo de sus necesidades relacionadas con su proceso educativo. Lo que más demandan las $N A J$ es el apoyo en la revisión de contenidos que no pudieron aprender en sus casas y escuelas, preparación de pruebas o de trabajos a través de instancias de encuentro lúdicas y participativas.

Por otra parte, están los CNAA, instancias de encuentro, aprendizaje y organización en torno a la solución de conflictos y exigibilidad de derechos, las que ocurren una vez por semana, en formato de asambleas en siete espacios territoriales diferentes (cuatro en Independencia, dos en Santiago y uno en Quinta Normal), emergiendo representantes de cada uno de los territorios (vocerxs), quienes se reúnen todos los martes en su respectiva Reunión de Vocerías. Espacio en el cual se organizan distintas actividades de exigibilidad, desarrollando instancias formativas relacionadas con sus derechos, así como manifestaciones, marchas, pasacalles (entre otras), o la redacción de cartas y declaraciones presentadas en instancias públicas.

Además, de manera transversal, se desarrollan instancias dentro de todos los espacios de acompañamiento, tanto en la ELSF como en los CNAA, destacando los momentos de ludoteca y los espacios de acogida. En relación con la ludoteca, se trata de acciones en general de tipo lúdicas, con fomento de la colectividad, el respeto, la cohesión grupal, la resolución dialogante de sus diferencias, recreación y la no violencia, ocurriendo principalmente al inicio de cada taller. Por su parte, los espacios de acogida son instancias donde las $N A J$ expresan sus emociones, experiencias y preocupaciones. Con ello, se construye un espacio de confianza y contención emocional donde es posible para las $N A J$ reportar sus vulneraciones de derechos, recibir orientación frente a las mismas y profundizar temas de interés. Todo esto se puede ver reflejado a través de los hitos y metodologías que serán abordados a continuación. 


\section{LA HISTORIA, LA MEMORIA Y PROCESOS DE ORGANIZACIÓN DE LAS NIÑECES: SALTANDO TORNIQUETES}

Para poder responder a nuestra pregunta, comenzaremos dando cuenta de lo ocurrido en el Carnaval Sin Fronteras 2019, siendo un pasacalle realizado desde el 2008 en el barrio La Chimba, organizado por las NAJ del CSF y la Comunidad Sin Fronteras, convocando diferentes muestras artístico-culturales andino-amazónicas.

El Carnaval nace porque las $N A J$ exigen respeto por su cultura, lugar de origen y la no discriminación. Las reglas del Carnaval son definidas por las $N A J$ y son compartidas con lxs directorxs de las agrupaciones musicales y danzantes, dejando claridad de que este es un espacio de respeto, libre de humos, drogas y alcohol. Se da énfasis en el carácter coparticipativo, político e intergeneracional del encuentro.

El Carnaval Sin Fronteras 2019 contó con más de cuatro mil participantes, incluyendo músicxs, danzantes y asistentes. Como todos los años, la obertura fue realizada por las vocerías del CSF, quienes leen una carta dirigida «al presidente de la república, a los diputados y senadores, a los medios de comunicación, a las instituciones y organizaciones públicas y privadas, a la comunidad, a nuestras familias, a los tíos y tías del Colectivo Sin Fronteras y a todos quienes debiesen ser nuestros garantes de derechos" 21 .

En dicha carta se realizan una serie de demandas, especificando que: «Queremos una educación intercultural para todos los niños y niñas sin importar su origen, raíz, raza y costumbres. Y por último, queremos una ley migratoria justa, que no discrimine y se preocupe por los derechos de todas las personas que

${ }^{21}$ Extracto de la carta leída al inicio del Carnaval Sin Fronteras 2019. 
migran, especialmente de los niños y niñas ${ }^{22}$. Se evidencian sus preocupaciones ante la contingencia nacional y sus implicancias en el tejido social, siendo fiel reflejo de sus procesos de participación y sus capacidades de asociatividad, autogobierno, autogestión y agencia colectiva propia de las $N A J$.

Antes de la revuelta social y durante todo el 2019, la violencia en la educación se identificó como un problema y se buscó interpelar a la comunidad, a la familia y la escuela a través de acciones de exigibilidad de derechos en espacios públicos como el frontis del CSF, el Centro Cultural Estación Mapocho, un centro comercial de la comuna de Independencia, la radio Juan Gómez Milla y revista Sur, en su programa Chile a Todo Color.

Cabe resaltar que durante la revuelta social —que se dio inicio a partir de octubre de 2019-, la violencia y represión ejercida por parte del Estado y la policía fue algo vivido de una manera particularmente cercana por las $N A J$. Esto ilustrado por dos situaciones de violencia policial sufrida por dos niños del CSF, cuando uno de ellos fue agredido físicamente durante las evasiones del metro (previo al 18 de octubre) y un segundo que recibió perdigones en sus piernas y $\operatorname{cara}^{23}$.

Estas dinámicas de violencia motivaron aún más la organización de las Asambleas Populares de Niñeces y Adolescencias de Quinta Normal y Casona Barnechea, las cuales tomaron lugar el 8 y 30 de noviembre, respectivamente. Estas instancias respondieron al contexto político y social que se vivía en aquellos días, sumado a las lógicas adultocéntricas que postergaron las voces de las $N A J$ de este proceso, siendo que lxs propixs secundarixs fueron quienes

\footnotetext{
${ }^{22}$ Idem.

${ }^{23}$ Ambas situaciones fueron denunciadas a la Defensoría de la Niñez y su proceso jurídico sigue en curso.
} 
impulsaron la revuelta popular. En el siguiente cuadro se pueden observar algunos de los acuerdos llegados en dichas asambleas.

\section{Cuadro 1. ACuerdos de las Asambleas Populares de las $N A J$ DEL 8 Y 30 DE NOVIEMBRE}

\begin{tabular}{|c|c|c|}
\hline $\begin{array}{c}\text { ¿Cómo nos hemos sentido } \\
\text { desde el inicio del } \\
\text { «estallido social»? }\end{array}$ & $\begin{array}{c}\text { ¿Qué podemos hacer } \\
\text { como niños y niñas para } \\
\text { mejorar esto? }\end{array}$ & $\begin{array}{c}\text { ¿Crees que NNA tienen } \\
\text { derecho a manifestarse y } \\
\text { por qué? }\end{array}$ \\
\hline $\begin{array}{l}\text { «Liberadx, enojadx, } \\
\text { impotencia» } \\
\text { «Nos sentimos } \\
\text { desprotegidxs por la } \\
\text { policía» } \\
\text { «Miedo, terror, alegría y } \\
\text { rabia» } \\
\text { «Fome igual, porque } \\
\text { tuvimos que hacer esto } \\
\text { para que el Estado nos } \\
\text { tomara en cuenta» } \\
\text { «Bien, porque la gente se } \\
\text { manifiesta por sus } \\
\text { derechos y abusos no } \\
\text { respetados» }\end{array}$ & $\begin{array}{l}\text { «Compartir lo que } \\
\text { sentimos con los demás» } \\
\text { «Descargar nuestro enojo } \\
\text { en modo de } \\
\text { manifestación» } \\
\text { «Exigir nuestros } \\
\text { derechos» } \\
\text { «Hay que hacer amigxs» } \\
\text { «Démonos más abrazos, } \\
\text { ¡muchos abrazos!» } \\
\text { «Manifestándose de forma } \\
\text { pacífica» } \\
\text { «Informar a más niños y } \\
\text { niñas de lo que está } \\
\text { pasando» }\end{array}$ & $\begin{array}{l}\text { «Sí, para hacer valer } \\
\text { nuestros derechos» } \\
\text { «Sí, porque nosotrxs } \\
\text { comenzamos el } \\
\text { movimiento y es a } \\
\text { nosotrxs a quienes más } \\
\text { violentan» } \\
\text { «Todxs tenemos libertad } \\
\text { de expresión» } \\
\text { «Pedimos un país más } \\
\text { social, más equitativo» } \\
\text { «Sí, porque estamos } \\
\text { luchando por nuestros } \\
\text { derechos, también porque } \\
\text { somos humanos y no } \\
\text { alienígenas }{ }^{24} »\end{array}$ \\
\hline
\end{tabular}

Fuente: Elaboración propia, a partir de las notas de campo del 8 y 30 de noviembre.

A raíz de este proceso, hubo claridad de las consignas co-construidas por la Comunidad Sin Fronteras, destacando y exigiendo dignidad humana; justicia social y buen vivir; educación gratuita de calidad, no sexista e intercultural; vida en familia; migración como derecho; Constitución política plurinacional; aumento salario mínimo; nacionalización de los recursos naturales; pensiones dignas; salud de calidad sin privatización; y el derecho a la vivienda.

Siguiendo estos procesos y buscando reivindicar y respetar los derechos humanos, se organiza el Carnaval Dignidad Sin Fronteras 2020, con la intención de conmemorar las violaciones a los derechos humanos y visibilizar los crímenes

\footnotetext{
${ }^{24}$ Haciendo alusión a los dichos por la primera dama Cecilia Morel en su audio filtrado.
} 
de lesa humanidad cometidos durante la revuelta social de octubre. Dicha instancia programada para abril de 2020 no se pudo concretar por la nueva crisis sociosanitaria.

\section{DE UNA CRISIS SOCIOPOLÍTICA A UNA CRISIS SANITARIA}

La crisis sociopolítica iniciada el 18 de octubre de 2019 en Chile vino a marcar una pauta en los objetivos que se habían propuesto para el 2020. Sin embargo, la crisis sanitaria exigió realizar cambios en la planificación anual de las actividades del CSF, continuando con la propuesta política de las $N A J$, a pesar de la pandemia.

Los primeros meses de la crisis sanitaria exigieron generar respuestas a las necesidades más urgentes de las familias, por lo que se levanta una serie de espacios que responden a iniciativas colaborativas y solidarias en los diferentes territorios que acompaña el CSF. Dentro de estas realidades, frente al hambre, el hacinamiento, la falta de acceso a derechos y la extrema vulnerabilidad, la organización y colaboración entre las familias deja de verse como una opción y se transforma en una necesidad para hacer frente a la crisis sanitaria.

En este sentido, habiendo identificado los graves efectos que venía teniendo la pandemia sobre la comunidad, se inicia la campaña «Solidaridad Sin Fronteras», entregando apoyo en alimentos e insumos de limpieza de manera quincenal a las familias que más lo necesitaron. Paralelamente, se conforman un total de cinco ollas comunes (cuatro en Independencia y una en Santiago), llegando a un total de 83 familias, donde 86 son NAJ y 169 adultxs, siendo 255 personas las que recibieron almuerzos diarios de lunes a viernes ${ }^{25}$.

Si bien las ollas comunes, los Comprando Juntos y los diferentes apoyos

${ }^{25}$ La campaña «Solidaridad Sin Fronteras» se mantuvo desde mayo a diciembre de 2020. 
a las familias más golpeadas buscaban satisfacer las necesidades más urgentes, estas también se transformaron en espacios de contención que respondieron a las demandas emocionales de las familias. Así mismo, se generaron instancias lúdicas virtuales que incluyeron bingos y Encuentros de Talentos para propiciar el sentido de comunidad a través de principios de solidaridad, afectividad y apoyo mutuo.

\section{ASAMbleas VIRTUALES 2020: NOS ORgANizAMOS CON O SIN PANDEMIA}

A raíz del compromiso asumido en las asambleas durante la revuelta social de mantener espacios estables de organización, se crean instancias de asambleas generales que convocaron a la totalidad de las NAJ del CSF, de todos sus espacios.

Estas asambleas se realizaron de manera virtual, teniendo diferentes finalidades a lo largo del segundo semestre de 2020, las cuales serán resumidas a continuación.

\subsection{ASAMblea Virtual del 30 DE JULio:} RESCATANDO LAS VOCES DE LAS NIÑECES Y ADOLESCENCIAS EN CONTEXTO DE PANDEMIA

La posibilidad de concretar una asamblea virtual se transformó en una realidad, contando con la participación de veintiocho NAJ de distintos espacios del CSF. Esta asamblea tuvo la particularidad de centrarse en la emocionalidad y los sentires como principales preocupaciones durante los primeros meses de pandemia. 
CuAdro 2. ACuerdos de la ASAmblea Virtual del 30 DE JUlio

\begin{tabular}{|c|c|c|}
\hline $\begin{array}{c}\text { ¿Cómo nos hemos sentido } \\
\text { como niñxs y adolescentes } \\
\text { en la situación de } \\
\text { pandemia que estamos } \\
\text { viviendo? }\end{array}$ & $\begin{array}{c}\text { ¿Qué le parece/qué } \\
\text { comentan como niñxs y } \\
\text { adolescentes sobre lo que } \\
\text { estamos viviendo en } \\
\text { pandemia? (Lo bueno y lo } \\
\text { malo)? }\end{array}$ & $\begin{array}{l}\text { ¿Qué acciones concretas } \\
\text { realizaremos como niñxs } \\
\text { y adolescentes sobre lo } \\
\text { que estamos viviendo en } \\
\text { la situación de pandemia? }\end{array}$ \\
\hline $\begin{array}{l}\text { «Algo nuevo, sofocante y } \\
\text { agobiante» } \\
\text { «Me he sentido con } \\
\text { mucha ansiedad. Al } \\
\text { principio tenía ganas de } \\
\text { comer, y ahora todo lo } \\
\text { contrario, como cada vez } \\
\text { menos» } \\
\text { «Decepción de que las } \\
\text { autoridades no hayan } \\
\text { tomado buenas } \\
\text { decisiones» } \\
\text { «Frustrada por la } \\
\text { conectividad y tareas» } \\
\text { «Temor a perder algún ser } \\
\text { querido» }\end{array}$ & $\begin{array}{l}\text { «La salud en Chile es un } \\
\text { asco» } \\
\text { «Las soluciones con } \\
\text { bonos que ha dado el } \\
\text { gobierno son ridículas, } \\
\text { pues los que más lo } \\
\text { necesitan no tienen } \\
\text { beneficios» } \\
\text { «La clase media no existe, } \\
\text { existe gente rica y pobres } \\
\text { como nosotrxs» } \\
\text { «He pasado por momentos } \\
\text { en donde dan esas crisis } \\
\text { existenciales en donde me } \\
\text { pregunto por qué estoy } \\
\text { viva, qué vengo a hacer al } \\
\text { mundo» }\end{array}$ & $\begin{array}{l}\text { «Enseñarles a nuestros } \\
\text { padres y madres sobre } \\
\text { acciones de autocuidado» } \\
\text { «Decirles a los colegios } \\
\text { que las casas no son un } \\
\text { lugar en donde una esté } \\
\text { acostumbrada a hacer } \\
\text { clases. Cuando uno llega a } \\
\text { la casa es para relajarse» } \\
\text { «Seguir con las juntas, } \\
\text { hablando, ya que quita } \\
\text { una carga de encima y nos } \\
\text { apoyamos entre todos» } \\
\text { «iNO PARAR!» }\end{array}$ \\
\hline
\end{tabular}

Fuente: Elaboración propia, a partir de las notas de campo del 30 de julio.

\subsection{ASAMBLEA VIRTUAL DEL 25 DE SEPTIEMBRE}

Luego de haber detectado un deterioro continuo de la salud mental y emocional de las NAJ, así como el aumento de la desigualdad en la educación, se optó por que la segunda asamblea virtual fuera en torno a la salud mental, las relaciones en pandemia y el acceso a la educación. Reflexiones que se presentan a continuación: 
CUAdRO 3. ACUERdos DE LA ASAMBleA VirTUAL DEL 25 DE SEPTIEMBRE

\begin{tabular}{|c|c|c|}
\hline $\begin{array}{c}\text { ¿Tenemos acceso a la } \\
\text { educación? }\end{array}$ & $\begin{array}{c}\text { ¿Cómo hemos enfrentado } \\
\text { la pandemia? }\end{array}$ & $\begin{array}{c}\text { ¿Nos hemos sentido mejor } \\
\text { por estar con nuestras } \\
\text { familias? }\end{array}$ \\
\hline $\begin{array}{l}\text { «La educación de calidad } \\
\text { normalmente se paga» } \\
\text { «Existen desigualdades al } \\
\text { estudiar presencial y } \\
\text { virtualmente» } \\
\text { «La educación no cumple } \\
\text { con los requisitos para que } \\
\text { las personas se desarrollen } \\
\text { de manera integral» } \\
\text { «Hay acceso para todxs, } \\
\text { pero no todxs tenemos el } \\
\text { mismo tipo de educación. } \\
\text { No todxs podemos } \\
\text { aprender de la misma } \\
\text { manera, todo depende del } \\
\text { colegio y los ingresos» }\end{array}$ & $\begin{array}{l}\text { «Ansiedad» } \\
\text { «Feliz por estar con mi } \\
\text { familia» } \\
\text { «Apoyo de familia y } \\
\text { amigos» } \\
\text { «Me siento mejor en la } \\
\text { casa que en la escuela» } \\
\text { «Me desahogo llorando» } \\
\text { «Al dormir se nota, un } \\
\text { cansancio que no } \\
\text { notamos, hay muchas } \\
\text { preocupaciones» } \\
\text { «Escuchando música» } \\
\text { «Tocar música y cantar } \\
\text { para alegrarse» } \\
\text { «Aprendiendo a hacer } \\
\text { cosas nuevas» }\end{array}$ & $\begin{array}{l}\text { «Hay peleas, pero son } \\
\text { pasajeras» } \\
\text { «Concretamente, con } \\
\text { apapachos, abrazos, afecto } \\
\text { físico» } \\
\text { «Quiero que mi familia se } \\
\text { vaya a trabajar» } \\
\text { «Hemos conversado } \\
\text { mucho, es bueno que se } \\
\text { sepan las cosas» } \\
\text { «Me he sentido muy feliz } \\
\text { al estar con mis padres, } \\
\text { más seguro, relajado» } \\
\text { «Me preocupa cuando mis } \\
\text { padres deben salir» } \\
\text { «Estamos más unidxs, } \\
\text { pero tenemos más } \\
\text { discusiones» }\end{array}$ \\
\hline
\end{tabular}

Fuente: Elaboración propia a partir de las notas de campo del 25 de septiembre

A pesar de las dificultades dadas por el contexto (falta de aparatos tecnológicos y conectividad), es relevante señalar la capacidad de readaptación por parte de las NAJ y Comunidad Sin Fronteras al levantar instancias de organización que permitieron el encuentro, fortalecer el vínculo entre $N A J$ y su comunidad, siendo un espacio de contención emocional y de reflexión acorde a la crisis sociosanitaria. 


\section{ENCUENTROS INTERNACIONALES}

Luego de una serie de instancias internas entre todas las $N A J$ del CSF, surge la necesidad de encontrarse con otras $N A J$ organizadas, con la intención de que sus demandas y consignas tomen más fuerza, sumando distintas experiencias de intercambio, con miras a acciones concretas de promoción y exigibilidad de sus derechos. A continuación, algunas de ellas.

\subsection{ENCUENTRO LATINOAMERICANO POR EL DERECHO A LA EDUCACIÓN, 12 Y 13 DE OCTUBRE DE 2020}

Se da la oportunidad de ser parte de una instancia internacional que convocó a juventudes de gran parte del Abya Yala ${ }^{26}$, con representantes de Colombia, Perú, México, Honduras, Argentina, Guatemala, República Dominicana, Venezuela, Bolivia y Chile, siendo representado por tres jóvenes, dos de ellos voceros del CSF.

Este encuentro convocó para tratar tres temas, a) educación decolonial y las resistencias educativas y culturales desde los territorios; b) identidades en situación de migración, desarraigo y desplazamiento; y finalmente c) educación $\mathrm{y}$ bienestar socioemocional de los pueblos originarios, afros, campesinos y urbano populares en época de pandemia.

\footnotetext{
${ }^{26}$ Se reconoce con este nombre originario al territorio que abarca desde México a países andinos. Apunta a un territorio sin límites ni fronteras y habitado por pluralidad de comunidades. Su nombre refiera a «tierra viva», «tierra de florecimiento», «tierra madura». Nuestra intención es dar cuenta de la pluralidad cultural, de historias, de saberes, que en común tienen el signo de la reciprocidad, de una relación estrecha con la tierra y, por consiguiente, de buenos vivires.
} 
En el siguiente cuadro, se pueden observar las preguntas que trabajaron las $N A J$ del CSF de manera previa, y que fueron movilizadas por las vocerías para dicho encuentro. A continuación, algunas de las ideas propuestas.

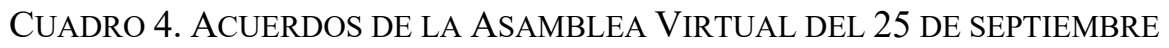

\begin{tabular}{|c|c|c|}
\hline $\begin{array}{c}\text { ¿Cómo la educación en } \\
\text { comunidades urbano- } \\
\text { populares resiste a la } \\
\text { educación del norte global } \\
\text { u occidental? }\end{array}$ & $\begin{array}{c}\text { ¿Cómo defienden su } \\
\text { identidad las comunidades } \\
\text { urbanas migrantes, en } \\
\text { desplazamiento o } \\
\text { desarraigo? }\end{array}$ & $\begin{array}{l}\text { ¿Cómo ha afectado la } \\
\text { pandemia a la educación } \\
\text { de las comunidades } \\
\text { urbanas marginadas? }\end{array}$ \\
\hline $\begin{array}{l}\text { «Incorporando la } \\
\text { emocionalidad en los } \\
\text { procesos» } \\
\text { «Aparte de aprender de } \\
\text { forma eurocentrista, } \\
\text { aprendemos de otras } \\
\text { formas que no nos } \\
\text { imponen» } \\
\text { «Incorporando el arte y la } \\
\text { cultura, el juego y la } \\
\text { recreación» } \\
\text { «Compartimos nuestras } \\
\text { ideas en grupo y } \\
\text { mantenemos espacios } \\
\text { estables de organización» }\end{array}$ & $\begin{array}{l}\text { «Manifestándonos, } \\
\text { haciendo muestras } \\
\text { artístico-culturales» } \\
\text { «Interpelando a distintos } \\
\text { garantes de derechos } \\
\text { (Estado/diputados/senador } \\
\text { es/medios de } \\
\text { comunicación/colegio)» } \\
\text { «Dando a conocer nuestra } \\
\text { identidad» }\end{array}$ & $\begin{array}{l}\text { «Cansador, aumento de } \\
\text { estrés, angustia e } \\
\text { incertidumbre académica» } \\
\text { «Mayor desigualdad, falta } \\
\text { de conectividad y } \\
\text { dispositivos tecnológicos» } \\
\text { «Problemas de salud } \\
\text { mental» }\end{array}$ \\
\hline
\end{tabular}

Fuente: Notas de campo del día 10 de octubre de 2020.

\subsection{ENCUENTRO DE NiÑECES Y AdOLESCENCIAS: VOCES LUCHANDO POR DIGNIDAD-MNNATSOP (PERÚ).,14 DE DICIEMBRE DE 2020}

Se genera un espacio de Facebook live preparado entre las vocerías del CSF y delegadxs del Movimiento Nacional de Niños, Niñas y Adolescentes Trabajadores Organizados del Perú (MNNATSOP), con la intención de dialogar e intercambiar experiencias en el marco de la semana de las culturas, por una migración digna, contando con la participación de cinco vocerxs y cinco 
delegadxs, siendo un espacio moderado por ellxs mismxs, libres de lógicas adultocéntricas.

Los temas que se abordaron en este espacio fueron relacionados con salud, educación, familia y trabajo digno de las NAJ. Este último punto fue una temática emergente dentro de las preocupaciones de las NAJ del CSF y que el MNNATSOP viene trabajando por más de 25 años.

A modo de síntesis, las ideas que surgieron en esta instancia de intercambio, y que fueron transmitidas por la revista Sur y las redes sociales de ambas organizaciones, se pueden resumir en el siguiente cuadro:

CUADRO 5. ACUERDOS DEL ENCUENTRO DE NIÑECES Y AdOLESCENCIAS: VOCES LUCHANDO POR DIGNIDAD

\begin{tabular}{|c|c|c|c|}
\hline Salud & Educación & Familia & Trabajo digno \\
\hline $\begin{array}{l}\text { «El gobierno no ha } \\
\text { ayudado» } \\
\text { «¿Dónde sacar } \\
\text { dinero con la } \\
\text { pandemia?» } \\
\text { «Necesitamos } \\
\text { sustentar a la } \\
\text { familia» } \\
\text { «Hay estrés } \\
\text { personal y de mis } \\
\text { padres» } \\
\text { «Aumentó la } \\
\text { depresión y la } \\
\text { incertidumbre } \\
\text { frente al futuro» }\end{array}$ & $\begin{array}{l}\text { «Aumentó la } \\
\text { desigualdad y la } \\
\text { deserción escolar» } \\
\text { «La educación no } \\
\text { les llega a todos» } \\
\text { «Pensamos que } \\
\text { sería más fácil pero } \\
\text { jamás consideraron } \\
\text { la pobreza» } \\
\text { «Las instituciones } \\
\text { públicas no tienen } \\
\text { interés en } \\
\text { solucionar estos } \\
\text { casos, somos el } \\
\text { futuro, pero ¿cómo } \\
\text { pretenden que lo } \\
\text { seamos si no } \\
\text { tenemos una } \\
\text { educación buena?» }\end{array}$ & $\begin{array}{l}\text { «Algo muy lindo, } \\
\text { pasar tiempo con } \\
\text { mis padres, pude } \\
\text { conocerlos más» } \\
\text { «Miedo, no } \\
\text { queríamos que la } \\
\text { pandemia nos } \\
\text { afectara» } \\
\text { «Pasó el tiempo y } \\
\text { los roces } \\
\text { aumentaron» }\end{array}$ & $\begin{array}{l}\text { «Nos dignifica } \\
\text { como personas, } \\
\text { nos permite poder } \\
\text { ayudar a la familia, } \\
\text { nos ayuda a } \\
\text { crecer» } \\
\text { «El trabajo para } \\
\text { crecer y dignificar, } \\
\text { con el necesario } \\
\text { acompañamiento } \\
\text { de las autoridades» } \\
\text { «Las autoridades } \\
\text { dicen que los niños } \\
\text { están atentando } \\
\text { contra su salud» } \\
\text { «Existe pobreza y } \\
\text { no hay ayuda» }\end{array}$ \\
\hline
\end{tabular}

Fuente: Elaboración propia, a partir de notas de campo del día 14 de diciembre de 2020. 
A raíz de estas instancias de intercambio con organizaciones de otros países y aprovechando las posibilidades que brinda la virtualidad, las $N A J$ del CSF se proponen generar un encuentro internacional que convoque a una pluralidad de organizaciones de $N A J$ de diferentes partes del Abya Yala.

\section{ENCUENTRO INTERCULTURAL 2021}

A raíz de la necesidad de encontrarse con otras NAJ, se da el 1er Encuentro Intercultural de Niñeces y Adolescencias en el marco del Carnaval Dignidad Sin Fronteras 2021, contando con la participación de diecisiete NAJ de Chile, Perú, Haití y México ${ }^{27}$.

27 Dicho encuentro se encuentra disponible en el siguiente link: https://fb.watch/6rlsaSaCFI/ 
CUADRO 6. ACUERDOS DEL ENCUENTRO INTERCULTURAL 2021

\begin{tabular}{|c|c|c|}
\hline $\begin{array}{l}\text { ¿Sienten que se respetan } \\
\text { las culturas en su } \\
\text { comuna/ciudad/país? }\end{array}$ & $\begin{array}{l}\text { ¿En qué y cómo nos } \\
\text { afectó la pandemia? }\end{array}$ & $\begin{array}{l}\text { Como niños, niñas y } \\
\text { adolescentes: ¿se respetan } \\
\text { nuestros derechos? }\end{array}$ \\
\hline $\begin{array}{l}\text { «Nos están oprimiendo y } \\
\text { negando nuestro derecho a } \\
\text { expresarnos» } \\
\text { «Se enseña a lxs niñxs a } \\
\text { discriminar» } \\
\text { «Me decían que yo no } \\
\text { tenía opinión porque este } \\
\text { era su país y yo era } \\
\text { migrante, sin derecho a } \\
\text { hablar y opinar, me quedé } \\
\text { un poco traumada y ahora } \\
\text { no participo mucho» } \\
\text { «Hay muchos países } \\
\text { donde se dan discursos de } \\
\text { odio hacia los migrantes» }\end{array}$ & $\begin{array}{l}\text { «En un principio no pensé } \\
\text { que podía llegar hasta } \\
\text { acá» } \\
\text { «Por lo que he visto, } \\
\text { también el tema de salud } \\
\text { mental nos ha afectado } \\
\text { mucho, trastornos } \\
\text { alimentarios, estar } \\
\text { encerrados, delirios de } \\
\text { persecución al salir a la } \\
\text { calle» } \\
\text { «Cuando la pandemia } \\
\text { termine se hará notar } \\
\text { cómo afectó a nuestra } \\
\text { salud» }\end{array}$ & $\begin{array}{l}\text { «Desde el } 18 \mathrm{O} \text {, el } \\
\text { gobierno ha mostrado que } \\
\text { no le interesa nuestra } \\
\text { integridad física, } \\
\text { emocional, educación, } \\
\text { solo le interesa su riqueza } \\
\text { y mantenerse en el poder» } \\
\text { «La PTU se vuelve un } \\
\text { capricho del gobierno por } \\
\text { no darnos una buena } \\
\text { educación desde un } \\
\text { principio» }\end{array}$ \\
\hline \multicolumn{3}{|c|}{ Propuestas } \\
\hline $\begin{array}{l}\begin{array}{l}\text { «Como pueblos, } \\
\text { estudiantes, } \\
\text { solo migrantes, } \\
\text { podemos }\end{array} \\
\text { organizarnos en nuestros } \\
\text { liceos, territorios, juntas de } \\
\text { vecinos y asambleas } \\
\text { territoriales» } \\
\text { «Exponer nuestras ideas, } \\
\text { reorganizar nuestras } \\
\text { propuestas y plantearlas } \\
\text { ante todos con un } \\
\text { representante nuestro» }\end{array}$ & $\begin{array}{l}\text { «Pedirles a los gobiernos } \\
\text { que apoyen a lxs NNA con } \\
\text { aparatos tecnológicos, no } \\
\text { pueden entrar a las clases y } \\
\text { tampoco tienen cómo } \\
\text { entrar» } \\
\text { «Pensando en la salud } \\
\text { mentaly quienes más estén } \\
\text { sufriendo, los mismos } \\
\text { gobiernos deberían } \\
\text { generar espacios de } \\
\text { atención» }\end{array}$ & $\begin{array}{l}\text { «Reclamar, manifestarse, } \\
\text { hacerle entender al Estado } \\
\text { que no todo lo que hacen es } \\
\text { suficiente, falta mucho por } \\
\text { hacer y necesitamos un } \\
\text { cambio constante» } \\
\text { «Hay que seguir luchando } \\
\text { por lo que creemos y exigir } \\
\text { nuestros derechos a los } \\
\text { garantes» }\end{array}$ \\
\hline
\end{tabular}

Fuente: Elaboración propia, a partir de notas campo del día 17 abril de 2021.

Como consecuencia de la experiencia anterior, las NAJ participantes del encuentro se proponen seguir fortaleciendo los vínculos forjados en pro de que sus análisis, reflexiones y propuestas sean considerados por la sociedad civil, estando dispuestos a realizar actividades de promoción, exigibilidad e incidencia en las discusiones y agenda pública. 


\section{REFLEXIONES}

La política antimigrante y racista ha significado un recrudecimiento de dinámicas de violencias sistemáticas, racismo y discriminación con las $N A J$ y sus familias, por lo que es necesario redoblar esfuerzos por contenerla y defender los avances alcanzados previamente, especialmente en materia de niñez.

La crisis sociosanitaria y política vino a tensionar las realidades vivenciadas por las $N A J$, acarreando consigo incertidumbre y la acentuación de las precariedades de la vida, generando malestares colectivos permanentes (Montero, 2004). La respuesta de la Comunidad Sin Fronteras y las NAJ se enmarca desde prácticas cotidianas, destacando principios de solidaridad, afectividad y apoyo mutuo, permitiendo acciones comunitarias e intergeneracionales (Cussiánovich, 2011) como respuesta concreta que funcionan desde lógicas políticas, económicas, sociales y culturales, dentro de las cuales podemos destacar las ollas comunes, el Carnaval Sin Fronteras virtual, Comprando Juntos, bingos y encuentros de talento en línea. De esta manera, nos encontramos frente a nuevas dinámicas sociales, donde la presencialidad se vuelve algo distante y el velar por la salud de manera integral, abarcando lo físico, mental, espiritual y emocional, un imperativo.

El proyecto político de las $N A J$ logra generar así espacios seguros ${ }^{28}$ (Fetner et al., 2016), de encuentro, interacción y discusión entre ellxs, resignificando la importancia y lo crucial, que es apostar por la interculturalidad (Walsh, 2010), la afectividad y la ternura (Cussiánovich, 2005) como la base para generar instancias de encuentro entre las $N A J$ y sus familias. En esa misma lógica, «un proceso participativo necesita del diálogo y de generar espacios de reflexión

\footnotetext{
${ }^{28}$ Entendiendo que estos espacios seguros de participación voluntaria se caracterizan por estar removidos del control directo de los grupos dominantes y adultocentristas, permitiendo que sus participantes aprendan sobre el respeto propio y desarrollen conexiones profundas, forjando una identidad grupal y promoviendo valores de cooperación y ciudadanía.
} 
crítica, que permita deconstruir significados que están arraigados en la cultura de las organizaciones y en los propios sujetos» (Muñoz, 2011: 123). Apostar por el coprotagonismo social y político de las $N A J$ (Cussiánovich, 2011) se vuelve decisivo en un contexto de crisis sociopolítica y sanitaria.

Por lo mismo, la propuesta política de las $N A J$ se basa en generar acción colectiva (Torres, 2009) desde nociones de ciudadanía crítica (González, 2015) y coprotagonismo (Cussiánovich, 2011), interpelando e invitando a la sociedad civil a cuestionar las dinámicas adultocentristas, para transitar hacia la coconstrucción de un nuevo modo de entablar las relaciones sociales intergeneracionales. De esta manera, su incorporación permitirá visibilizar el carácter político de las relaciones entre las $N A J$ y de ellxs con lxs adultxs, observando el modo en el cual participan en su propio autogobierno (González, 2015).

Si bien, la respuesta de las $N A J$ se basa en un enfoque de derechos, lo cierto es que comprenden la CDN como fiel reflejo de la cultura adultocéntrica, ya que se les ha excluido de manera histórica del debate y la posibilidad de incidir en las formas e implicancias de generar mecanismos de protección y exigibilidad de sus propios derechos (Morales y Magistris, 2018). Lo anterior se ha expresado en derechos que la CDN no resguarda, como el derecho al trabajo digno de las $N A J$, invisibilizando las realidades de niñeces que tienen la necesidad de trabajar para enfrentar la precarización de la vida a la que se ven enfrentadxs, por lo que han tenido que ocupar un rol para el sustento económico del hogar y de esta manera enfrentar las adversidades de la crisis sociosanitaria. Es así como se expresan malestares y violencias en las vidas de las $N A J$, en donde la CDN y el Estado de Chile quedan al debe a la hora de resguardar y proteger los derechos de las niñeces.

Es así, como las $N A J$ del $C S F$ han podido responder en primer lugar a través de sus propios espacios de organización, en donde el optar por un rol 
protagónico de sus procesos es posible, logrando así crear comunidades resilientes que tienen la capacidad de enfrentar sus malestares colectivos a través de reflexiones y acciones concretas. Por lo mismo y respondiendo a la pregunta ¿cuál es la respuesta que las $N A J$ desarrollan frente al contexto continuo de crisis sociopolítica y sanitaria?, es atingente resaltar la relevancia de mantener espacios permanentes de encuentro, tales como los espacios de acogida, rescatando la importancia de contar con instancias de contención emocional, así como de análisis y reflexión de la contingencia, siendo coherentes con la pedagogía de la ternura (Cussiánovich, 2005), la educación popular (Freire, 2005) y sus principios; asambleas generales, relevando la capacidad de asociatividad, autogobierno, agencia y de readaptación por parte de las $N A J$ y la Comunidad Sin Fronteras, que se ve reflejado en sus acciones para enfrentar las adversidades de la crisis sociosanitaria. El hecho de que estos espacios de organización propicien instancias de problematización a través del fortalecimiento de vínculos sociales y afectivos, permite el desarrollo de discusiones y acciones que evidencian las nociones de coprotagonismo (Cussiánovich, 2011), ciudadanía crítica y radical (González, 2015) entre las $N A J$ del CSF; y por último, los encuentros con otras $N A J$ permitieron el intercambio de experiencias y saberes, desde lo que conocemos como interculturalidad crítica (Walsh, 2010), fortaleciendo el desarrollo de vínculos, con el objetivo de desarrollar encuentros a futuro, así como también acciones de exigibilidad, promoción e incidencia en la agenda pública.

Así, la respuesta dada por las $N A J$ es una que pide romper las fronteras nacionales, crear comunidades haciendo uso de las nuevas tecnologías y recordando que, a través del afecto, la ternura, el amor y la emocionalidad, podemos generar comunidades resilientes capaces de organizarse en los peores escenarios de crisis. Procesos como estos nos llevan a comprender y situar el agenciamiento de los movimientos sociales de las NAJ, como renovadas experiencias de ciudadanía (Vargas, 2003), confluyendo en condiciones políticas 
y sociales estructurales, propias de la experiencia asociativa, las que no necesariamente responden a los parámetros tradicionales de organización, entendiendo la voluntad política, los movimientos sociales y la acción colectiva desde sus propios protagonistas como ciudadanxs que son parte del tejido social, desarrollando prácticas intergeneracionales, antiadultistas, antipatriarcales y antirracistas que permitan el desarrollo de una posibilidad práctica del buen vivir.

RECIBIDO: 1 DE JULIO DE 2021 ACEPTADO: 31 DE AGOSTO DE 2021

\section{BiBLIOGRAFÍA}

ABRAmovich, V. (2006). Una aproximación al enfoque de derechos en las estrategias y políticas de desarrollo. Revista de la CEPAL, 88, 35-50. Recuperado de: https://www.cepal.org/es/publicaciones/11102aproximacion-al-enfoque-derechos-estrategias-politicas-desarrollo

Alexgaias, A. (2014). El Manifiesto Antiadultista. Galiza: Distribuidora Anarquista Polaris.

Alfageme, E., Martínez, M. y Cantos, R. (2003). De la participación al protagonismo infantil. Madrid: Plataforma de Organizaciones de Infancia

Areyuna, B., Cabaluz, F. y Zurita, F. (2018). Educación popular y pedagogías críticas. Corrientes emancipadoras de la educación chilena. En A. Guelman, F. Cabaluz y M. Salazar, Educación Popular y Pedagogías críticas en América Latina y el Caribe. Buenos Aires: Clacso.

Cussiánovich, A. (2011). Hacia un nuevo contrato social por la dignidad de los niños y niñas. Lima: INFANT.

. (2010). Aprender la condición humana: ensayo sobre pedagogía de la ternura. Lima: Ifejant. Recuperado de: https://biblioteca.isauroarancibia.org.ar/aprender-la-condicion-humanaensayo-sobre-la-pedagogia-de-la-ternura/

Cussiánovich, A. (2005). Educando desde una pedagogía de la ternura. Lima: Ifejant. Recuperado de: https://enlafiladeatras.files.wordpress.com/2013/01/pedagogia-de-laternura-1.pdf 
Cussiánovich, A. y MÁRQuez, A. (2002). Hacia una participación protagónica de los niños, niñas y adolescentes. Save the Children Suecia-Oficina regional para América del Sur. [Versión digital] Recuperado de: https://www.sename.cl/wsename/otros/Hacia\%20una\%20participacion $\% 20$ protagonica- savethechildren.pdf

DEFENSORÍA DE LA NiÑEZ (2020). Informe Anual 2020. Derechos humanos de niños, niñas y adolescentes en Chile. Recuperado de: https://www.defensorianinez.cl/informe-anual/wpcontent/uploads/2020/11/ia2020.pdf

Facio, A. (2005). Feminismo, Género y Patriarcado. Academia. Revista sobre enseñanza sobre el derecho de Buenos Aires, 3(6), 259-294.

Fetner, T., Elafros, A., Bortolin, S. y Drechsler, C. (2016). Safe Spaces: Gay-Straight Alliances in High School. Recuperado de: https://doi.org/10.31235/osf.io/y6g82

Fundación Friedrich EBERT-ChILE (2017). Manual de Mitos y Realidades sobre la Migración en Chile. Santiago, Chile: Andros Impresores. Recuperado de: https://library.fes.de/pdf-files/bueros/chile/14453.pdf

GómEZ NASHIKI, A. (2013). Bullying: El poder de la violencia. Una perspectiva cualitativa sobre acosadores y víctimas en escuelas primarias de Colima. Revista mexicana de investigación educativa, 18(58), 839-870. Recuperado http://www.scielo.org.mx/scielo.php?script=sci_arttext\&pid=S1405$66662013000300008 \& \operatorname{lng}=$ es\&tlng=es

GonZÁLEZ, A. (2016). El desafío de una ciudadanía crítica en la infancia chilena. Desidades, $\quad 11, \quad 22-31 . \quad$ Recuperado de: http://pepsic.bvsalud.org/scielo.php?script=sci_arttext\&pid=S231892822016000200003\&lng=pt\&tlng=es.

GÜENDEL, L. (2000). La política pública y la ciudadanía desde el enfoque de los derechos humanos: la búsqueda de una nueva utopía. En S. SoTo (ed.), Política social: vínculo entre Estado y sociedad. San José: Fondo de Las Naciones Unidas para La Infancia/Flacso/Banco Mundial. Recuperado de: $\quad$ https://www.civilisac.org/civilis/wp-content/uploads/derechoshumanos-y-polc3adticas-pc3bablicas-guendel-1.pdf

LiEBel, M. (2016). La Declaración de Moscú sobre los Derechos del Niño (1918): un aporte desde la historia oculta de los Derechos de la Infancia. Educació Social. Revista d'Intervenció Socioeducativa, 62, 24-42.

Montero, M. (2004). Introducción a la psicología Comunitaria. Desarrollo, conceptos y procesos. Buenos Aires: Paidós Recuperado de: http://www.catedralibremartinbaro.org/pdfs/libro-montero-introducciona-la-psicologia-comunitaria.pdf 
Morales, S. y Magistris, G. (comps.) (2018). Niñez en Movimiento, del Adultocentrismo a la emancipación. Buenos Aires: Ternura Revelde.

MuÑoz, G. (2011). La democracia y la participación en la escuela: ¿cuánto se ha avanzado desde la normativa para promover la participación escolar? Revista de Estudios y Experiencias en Educación, 10(19), 107-129. Recuperado de: http://www.rexe.cl/19/pdf/196.pdf

Obach, A., Sadler, M. y Aguayo, F. (2011). Resultados del Proyecto Involucrando hombres jóvenes en el fin de la violencia de género. Intervención multipaís con evaluación de impacto. Caso chileno. Santiago de Chile: CulturaSalud/EME.

ONU (1989). Asamblea General, Convención sobre los Derechos del Niño, 20 de noviembre. United Nations, Treaty Series, vol. 1577, p. 3. Recuperado de: https://www.refworld.org.es/docid/50ac92492.html

QuiJano, A. (2011). Bien Vivir: entre el desarrollo y la des/colonialidad del poder. Ecuador Debate, 84, 77-88.

SAVE THE CHILDREN Suecia (2008). Promoción de la participación protagónica y significativa de los niños y adolescentes expuestos a la violencia. Recuperado de: https://resourcecentre.savethechildren.net/sites/default/files/documents/ 1426_0.pdf

Solís, S. (2003). El enfoque de derechos: aspectos teóricos y conceptuales. Recuperado de: http://www.ts.ucr.ac.cr/binarios/docente/pd-000133.pdf

TORRES, C. (2009). Acción Colectiva y Subjetividad. Un balance desde los estudios sociales. Revista Folios, 30, 51-74.

VAlVerde, F. (2004). Apuntes sobre enfoque de derechos. Asociación Chilena por Naciones Unidas (ACHNU).

VArgas, J. (2003). Teoría de la Acción Colectiva, Sociedad Civil y los Nuevos Movimientos Sociales en las formas de gobernabilidad en Latinoamérica. Espacio Abierto, 12(4), 523-537.

WALSH, C. (2010). Interculturalidad crítica y educación intercultural. Construyendo interculturalidad crítica, 75, 96. Recuperado de: https://www.uchile.cl/documentos/interculturalidad-critica-y-educacionintercultural_150569_4_4559.pdf

. (2009). Interculturalidad, estado, sociedad: luchas (de) coloniales de nuestra época. Quito: Universidad Andina Simón Bolívar/Ediciones Abya-Yala. Recuperado de: https:/www.academia.edu/35011983/ INTERCULTURALIDAD_ESTADO_SOCIEDAD_LUCHAS_DE_CO LONIALES_DE_NUESTRA_\%C3\% $\%$ PPOCA 